\title{
Reticulon1-C modulates protein disulphide isomerase function
}

\author{
P Bernardoni ${ }^{1,3}$, B Fazi ${ }^{1,3}$, A Costanzi ${ }^{1}$, R Nardacci ${ }^{2}$, C Montagna ${ }^{1}$, G Filomeni ${ }^{1}$, MR Ciriolo ${ }^{1}$, M Piacentini ${ }^{1,2,4}$ and F Di Sano ${ }^{*, 1,4}$
}

Endoplasmic reticulum (ER) is the primary site for the synthesis and folding of secreted and membrane-bound proteins. Accumulation of unfolded and misfolded proteins in ER underlies a wide range of human neurodegenerative disorders. Hence, molecules regulating the ER stress response represent potential candidates as drug targets for tackling these diseases. Protein disulphide isomerase (PDI) is a chaperone involved in ER stress pathway, its activity being an important cellular defense against protein misfolding. Here, we demonstrate that human neuroblastoma SH-SY5Y cells overexpressing the reticulon protein 1-C (RTN1-C) reticulon family member show a PDI punctuate subcellular distribution identified as ER vesicles. This represents an event associated with a significant increase of PDI enzymatic activity. We provide evidence that the modulation of PDI localization and activity does not only rely upon ER stress induction or upregulation of its synthesis, but tightly correlates to an alteration in its nitrosylation status. By using different RTN1-C mutants, we demonstrate that the observed effects depend on RTN1-C N-terminal region and on the integrity of the microtubule network. Overall, our results indicate that RTN1-C induces PDI redistribution in ER vesicles, and concomitantly modulates its activity by decreasing the levels of its $S$-nitrosylated form. Thus RTN1-C represents a promising candidate to modulate PDI function.

Cell Death and Disease (2013) 4, e581; doi:10.1038/cddis.2013.113; published online 4 April 2013

Subject Category: Neuroscience

Protein disulfide isomerases (PDIs) are endoplasmic reticulum (ER)-resident proteins. They catalyze the formation and reduction of disulfide bonds ${ }^{1}$ and they also act as chaperones, therefore, being an important cellular defense against protein misfolding.

In recent years, PDIs have been identified as novel therapeutic targets for the unfolded protein response (UPR) modulation. Recent studies report that several human disorders originate from dysfunctions in the ER and that regulation of some important UPR mediators, such as PDIs, may represent potential targets for modulating ER stress response. ${ }^{2}$

An increase of PDI activity could counteract protein inclusions formation, which are distinctive signs of neurodegenerative diseases. ${ }^{3,4}$ Consistent with this assumption, several reports suggest that PDI function has a crucial role in neuronal cell death, as it is able to attenuate neurotoxicity associated with the accumulation of aggregated proteins which is, in turn, responsible for neurodegenerative processes. Along this line, it has been suggested that PDI activity is impaired in amyotrophic lateral sclerosis (ALS), ${ }^{3}$ and PDI immunopositive inclusions have been found being part of neurofibrillary tangles in the brain of patients affected by Alzheimer's disease (AD). ${ }^{5}$

In the course of neurodegenerative diseases, neurons ultrastructure is altered and ER is damaged leading to the release of PDI out of the ER; interestingly, it has been recently suggested that reticulon (RTN) proteins may have a key role in these processes in particular in the context of ALS. ${ }^{6}$ Nevertheless, the molecular mechanism regulating these events are largely uncharacterized.

RTNs are membrane-bound proteins that have a uniquely conserved C-terminal domain named RHD and a variable $\mathrm{N}$-terminal region. ${ }^{7}$ In mammals, four genes have been identified, namely rtn1, 2, 3 and the neurite outgrowth inhibitor rtn4/nogo. RTNs have attracted particular interest due to their implication in different cellular processes, including ER stress induction and apoptosis. ${ }^{8}$ The pattern of RTNs localization in the ER, Golgi and plasma membrane strongly suggests their involvement in vesicle trafficking. Accordingly, it has been demonstrated that RTNs interact with several proteins regulating endo- and exocytosis processes. ${ }^{9,10}$ More recent studies have also involved RTNs in several CNS disorders, ${ }^{11-13}$ such as AD and ALS. ${ }^{8}$

\footnotetext{
${ }^{1}$ From the Department of Biology, University of Rome 'Tor Vergata', Via della Ricerca Scientifica, 00133 Rome, Italy and ${ }^{2}$ From the National Institute for Infectious Diseases IRCCS 'L. Spallanzani', Via Portuense, 00149 Rome, Italy

${ }^{*}$ Corresponding author: F Di Sano, From the Department of Biology, University of Rome 'Tor Vergata', Via della Ricerca Scientifica, 00133 Rome, Italy. Tel. + 39067259 4234; Fax + 39062023 500. E-mail: federica.di.sano@ uniroma2.it

${ }^{3}$ These authors contributed equally to this work.

${ }^{4}$ Co-corresponding authors.

Keywords: reticulon; protein disulfide isomerase; endoplamic reticulum

Abbreviations: RTN1-C, reticulon protein 1-C; PDI, protein disulfide isomerase; ER, endoplasmic reticulum; UPR, unfolded Protein response; ALS, amyotrophic lateral sclerosis; NFTs, neurofibrillary tangles; AD, Alzheimer's disease; RHD, reticulon homology domain; SNO-PDI, S-nitrosylated PDI; NOS, nitric oxide synthase; SOD1, superoxide dismutase 1; PD, Parkinson's disease; Tun, tunicamycin; TG, thapsigargin; Doxy, doxycycline; Noco, nocodazole; FBS, fetal bovine serum; DMSO, dimethyl sulfoxide; BSA, bovine serum albumin; PBS, phosphate-buffered saline; MMTS, methyl methanethiosulfonate

Received 20.2.13; accepted 28.2.13; Edited by G Melino
} 
Considering this context we decided here to investigate the role of reticulon protein 1-C (RTN1-C) on the intracellular localization and functional modulation of PDI. We demonstrated that RTN1-C overexpression induces a marked intracellular PDI redistribution, from a diffuse to a punctate pattern. We show that these structures are ER derivativevesicles and that PDI cellular relocalization is not the result of ER stress induction, but of the modulation of its $S$-nitrosylation status, which also significantly impacts on its enzymatic activity. We finally identified the $\mathrm{N}$-terminal domain of the RTN1-C as the putative domain involved in the modulation of these events.

\section{Results}

RTN1-C modulates PDI cellular localization. Considering the importance of both RTN1-C and PDI in neuronal cell homeostasis we decided here to investigate the functional relationship between the two proteins. We first verified whether RTN1-C was able to modulate PDI subcellular localization in SH-SY5Y human neuroblastoma cells. To this end, we transiently transfected this cell line with an expression vector coding for RTN1-C. We found that overexpression of RTN1-C induced a marked change in PDI distribution from a diffuse to a more punctate pattern (Figure 1a). Based on these findings we next characterized the RTN1-C effect on PDI intracellular localization by performing a time-course experiment using a tetracyclineinducible SH-SY5Y RTN1-C cell line (SH-SY5YRTN1-C $){ }^{14}$ Cells were treated with doxycycline (Doxy) for 3, 6, 18 and $24 \mathrm{~h}$ and analyzed for PDI localization by fluorescence microscopy (Figure 1b). Increase of RTN1-C expression levels were also confirmed by western blot analysis (Figure 1c). Results obtained showed that PDI punctate pattern became evident already after $6 \mathrm{~h}$ of RTN1-C induction, suggesting that PDI subcellular redistribution is a relatively early event in the ER-signaling pathway. After $18 \mathrm{~h}$, the number of cells showing PDI redistribution increases, along with the size of PDI dots (Figures $1 \mathrm{~b}$ and $\mathrm{d}$ ), indicating that the kinetics of PDI puncta formation temporally correlates with RTN1-C expression. It is worthwhile mentioning that this effect is specific for RTNs, as the overexpression of other ER resident proteins is unable to induce the same PDI relocalization. ${ }^{6}$

We then decided to deeply investigate the nature of the PDIcontaining puncta in order to identify their origin. To address this point, RTN1-C overexpressing cells were analyzed by electron microscopy. Figure 2 revealed the presence of electron-dense inclusions, and immunogold analysis, performed using anti-PDI and anti-calreticulin antibodies, indicated that these structures derived from the ER; in fact, the double immunolabeling showed that PDI colocalized with the ER marker calreticulin (Figure 2).

RTN1-C affects PDI activity. On the basis of the results obtained we then decided to investigate whether the RTN1C-mediated PDI relocalization has any effect on its enzymatic activity. We measured PDI activity following RTN1-C induction (Figure 3 and Supplementary Figure 1a ) according to Lovat et al. ${ }^{15}$ Interestingly, results obtained from these experiments show a significant increase of PDI enzymatic activity, which was particularly evident after $48 \mathrm{~h}$ from RTN1-C induction (Figure 3a).

Considering the key role played by both PDI and RTN1-C in ER stress induction, ${ }^{14}$ we wondered whether the observed changes in PDI activity were linked to perturbation in ER homeostasis. To assess this hypothesis, we performed PDI enzymatic assay on SH-SY5YRTN1-C cells treated with tunicamycin (Tun), a well-known ER stress inducer. The results obtained show that Tun is able to increase PDI activity (Figure 3c), thereby resembling the effect of RTN1-C overexpression. However, western blot analysis (Figure 3d) of PDI indicated that this event was mainly associated with an increase of its expression levels. We further confirmed these data by performing the same experiments (analysis of PDI expression and activity) using another ER stress inducing compound acting by a different mechanism, that is, thapsigargin (TG). Together the results obtained (Figure $3 f-h$ ) suggest that perturbation of ER homeostasis results in PDI activation, which is likely to be directly linked to an increase of its expression. Conversely, PDI concentration was not affected in RTN1-C overexpressing cells, suggesting that, in this case, the modulation of enzymatic activity of PDI is independent of its synthesis (Figures $3 d$ and e).

We then investigated PDI distribution in neuroblastoma cells treated with Tun by fluorescence microscopy. As expected, subcellular localization of the protein did not change in response to ER stress induction elicited by Tun administration (Figure 4), suggesting that the modulation of PDI enzymatic activity induced by RTN1-C is somehow dependent on its cellular redistribution. However, immunoprecipitation analyses excluded that the RTN1-C effect on PDI depends on a direct physical interaction between the two proteins (data not shown).

Changes in PDI activity are related to a different nitrosylation status of the enzyme. It is known that PDI activity is regulated by S-nitrosylation processes. ${ }^{4}$ In particular, S-nitrosylated PDI (SNO-PDI) is catalytically inhibited by reversible nitric oxide-mediated modification of the reactive cysteines present in the active sites of the enzyme. We then performed a biotin-switch assay to verify whether the RTN1-C-dependent modulation of PDI activity could rely upon changes of the nitrosylation status of this enzyme. Results shown in Figure 5 indicate that, in spite of the similar expression levels of PDI detected in SH-SY5Y ${ }^{\mathrm{RTN} 1-\mathrm{C}}$ and in the parental counterpart, the $S$-nitrosylated fraction of the protein differs significantly, with the RTN1-C overexpressing cell line showing a very limited amount of SNO-PDI. Interestingly, these results not only confirmed the above reported data on PDI activity (see Figure 3) and strongly indicated a positive modulation of PDI function by RTN1-C, but also are in line with our previous findings showing that RTN1-C expression negatively correlates with the inhibition of nitric oxide synthase (NOS). ${ }^{16}$

RTN1-C N-terminal region is involved in PDI redistribution. All RTNs share a C-terminal conserved domain (RHD) comprising two hydrophobic regions flanking a hydrophilic 
a
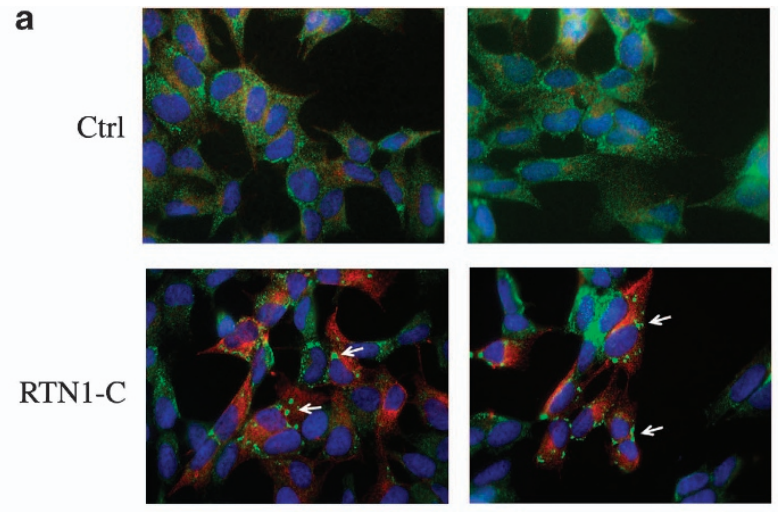

b

Doxy (hours)
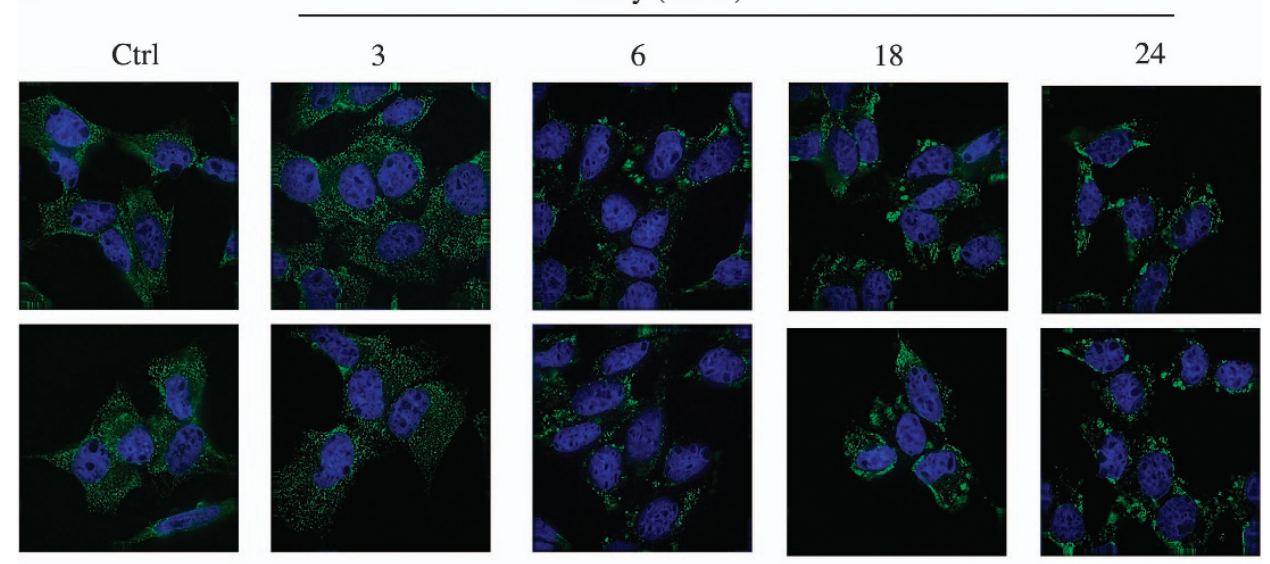

C
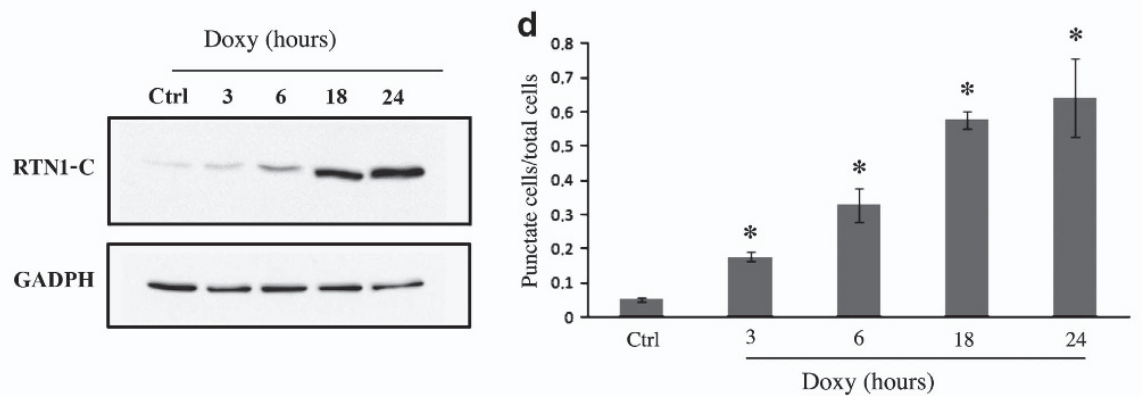

Figure 1 RTN1-C affects PDI subcellular distribution. (a) SH-SY5Y neuroblastoma cells were transfected for $24 \mathrm{~h}$ with an expression vector carrying the cDNA for RTN1-C or the vector alone (Ctrl) and analyzed by fluorescence microscopy. Cells were stained with anti-PDI (green) and anti-RTN1-C (red) antibodies, respectively. Nuclei were counterstained by using the fluorescent dye Hoechst 33342. Images are representative of three different experiments. Arrows: punctuated PDI. Original magnifications: $\times 100$. (b) SH-SY5Y ${ }^{\text {RTN1-C }}$ cells were induced with $1 \mu \mathrm{g} / \mathrm{ml}$ Doxy for 3, 6, 18 and $24 \mathrm{~h}$, stained with an PDI antibody (green) and analyzed by fluorescence microscope respect to untreated cells (Ctrl). Nuclei were labeled by using the fluorescent dye Hoechst 33342. Images are representative of three different experiments. Original magnifications: $\times 100$. (c) Alternatively, cells were lysed and analyzed by western blot for RTN1-C expression. GAPDH was used as loading control. The results shown are representative of three independent experiments. (d) SH-SY5YRTN1-C cells visualized in $\mathbf{b}$ were counted in at least ten different fields and the number showing puntuated PDI localization was compared with total cells. Results shown represent the means \pm S.D. of three independent determinations. *Student's $t$-test, $P<0.01$ for each condition versus control

loop, while the $\mathrm{N}$-terminal region of RTNs represents the variable part that is likely responsible for the specific biological function of each isoform. ${ }^{9,10,17,18}$ On the basis of the results obtained here, we investigated about the RTN1-C protein domain involved in the above-described phenomena. We generated different RTN1-C mutant proteins in particular we used two different mutant versions: the first with a single point mutation in the lysine 204 , able to inhibit RTN-mediated ER stress signaling, ${ }^{19}$ and the second carrying a deletion of the first nine amino acids in the $\mathrm{N}$-terminal region of the protein (Figure 6a). SH-SY5Y cells were transiently transfected with the mutated constructs and analyzed by fluorescence microscopy for PDI subcellular localization (Figure 6b). The expression levels of wild-type RTN1-C (WT) and different RTN1-C mutant proteins (RTN1-C K204 and RTN1-C- $\Delta \mathrm{N}$ ) were analyzed by western blot (Supplementary Figure 1b). Results shown in Figure $6 \mathrm{~b}$ show that, while the K204 mutant was still able to induce the $\mathrm{PDI}$ redistribution, the $\mathrm{N}$-terminal deleted mutant did not display the same effect. In particular, SH-SY5Y cells 


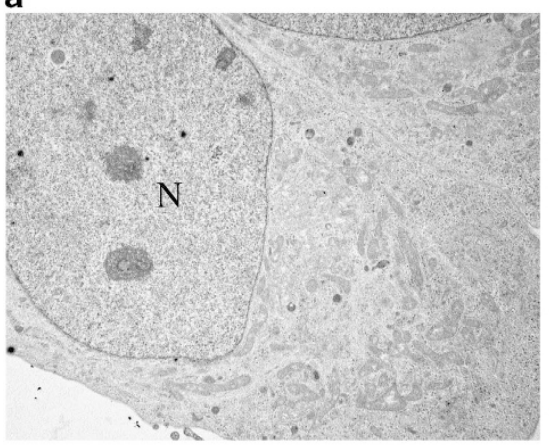

c

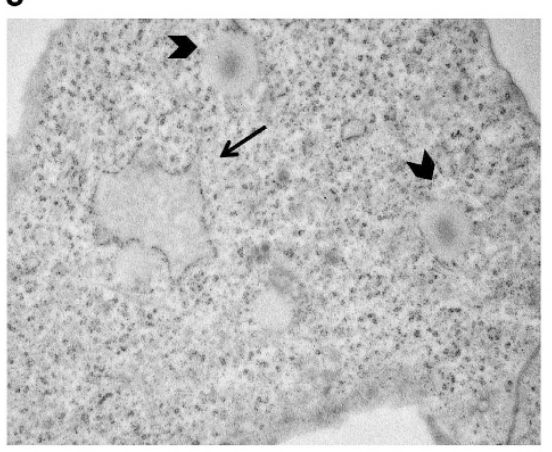

e

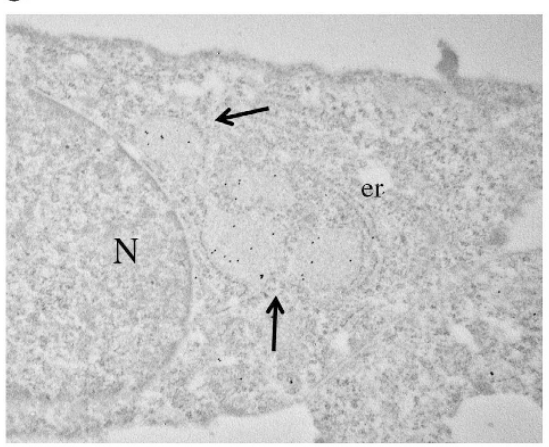

b

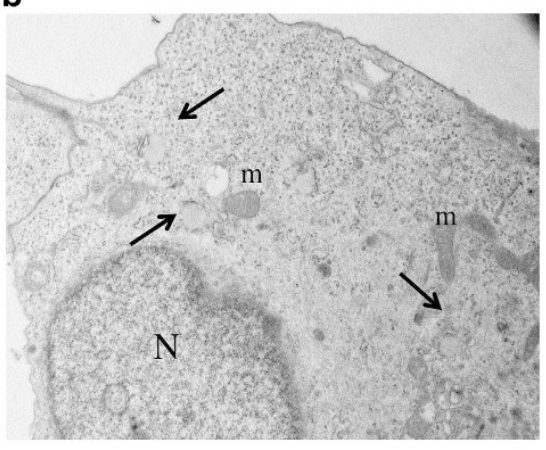

d

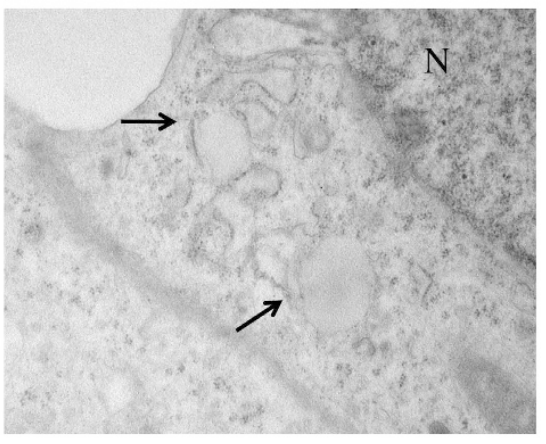

f

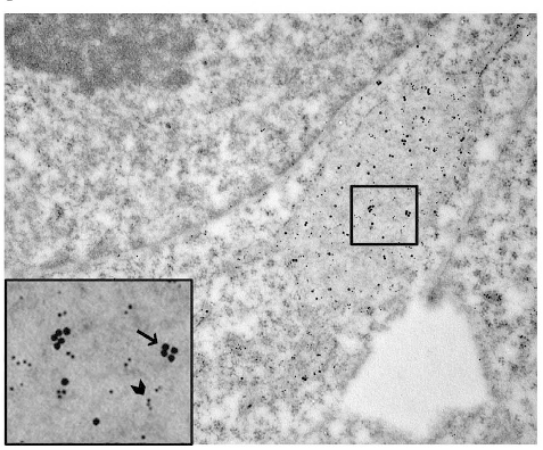

Figure 2 Ultrastructural analyses of RTN1-C overexpressing cells. SH-SY5YRTN1-C were left untreated (a) or treated with $1 \mu \mathrm{g} / \mathrm{ml}$ Doxy for $48 \mathrm{~h}(\mathrm{~b}-\mathrm{d})$ fixed in $2.5 \%$ glutaraldehyde and visualized by electron microscopy. Arrows indicate the accumulation of electron-dense material in the cytoplasm deriving from the ER; $m$, mitochondria; arrowheads indicate lipid droplets, the content of which is clearly different from the ER-derived inclusions. (e) Doxy-treated SH-SY5YRTN1-C were subjected to immunogold analysis with anti-PDI and anti-calreticulin antibodies, the latter of which was used as ER mark(er). Arrows indicate PDI accumulation in enlarged ER. (f) Detail of ER cisternae with a higher magnifications of the boxed area shown as inset at the bottom left. Anti-PDI ( $15 \mathrm{~nm}$, arrows) and anti-calreticulin ( $5 \mathrm{~nm}$, arrowheads). $\mathrm{N}$, nucleus. Original magnifications: a: $\times 4.400, \mathrm{~b}: \times 7.000, \mathrm{c}: \times 12.000, \mathrm{~d}, \mathrm{e}: \times 20.000, \mathrm{f:} \times 30.000$

transfected with the RTN1-C- $\Delta \mathrm{N}$ construct display a PDI localization similar to the diffuse pattern detected in control cells. On the basis of these results, we subsequently analyzed PDI enzymatic activity in response to the RTN1-C $\mathrm{N}$-terminal deletion. Figure 7 shows that there was no significant difference of PDI activity measured in RTN1-C- $\Delta \mathrm{N}$ overexpressing cells respect to their controls, confirming the functional relationship between PDI redistribution and its enzymatic function.

PDI redistribution depends on microtubules network organization. ER is highly dynamic and undergoes continuous fusion processes between tubules and sheets, and cytoskeleton is deeply involved in the distribution and formation of ER tubules. PDI distribution is strongly affected by the disruption of microtubule organization due of its strong influence on ER structure and integrity. ${ }^{20}$ Given the role of RTN proteins in membrane shaping and vesicle trafficking, we evaluated whether RTN1-C effect on PDI redistribution may depend on the integrity of microtubule network and stability. To this aim PDI subcellular localization was monitored in SH-SY5Y ${ }^{\text {RTN1-C }}$ cells after Doxy administration in the presence of nocodazole (Noco), a well-known microtubule-disassembling compound. Results shown in Figure 8 demonstrate that depolymerization of microtubules was able to completely abrogate RTN1-C-mediated PDI redistribution, suggesting that microtubules network has a key role in directing diffuse to punctuate distribution of PDI. 


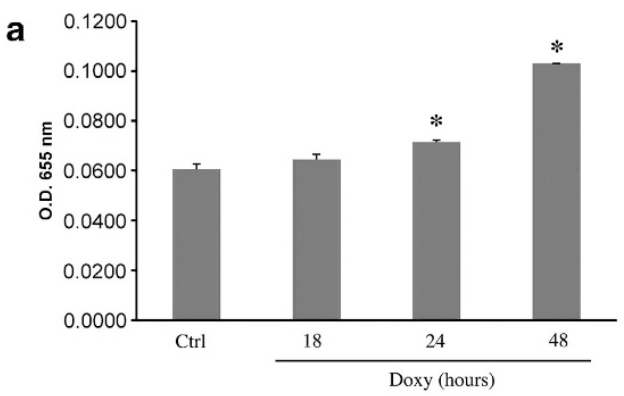

b
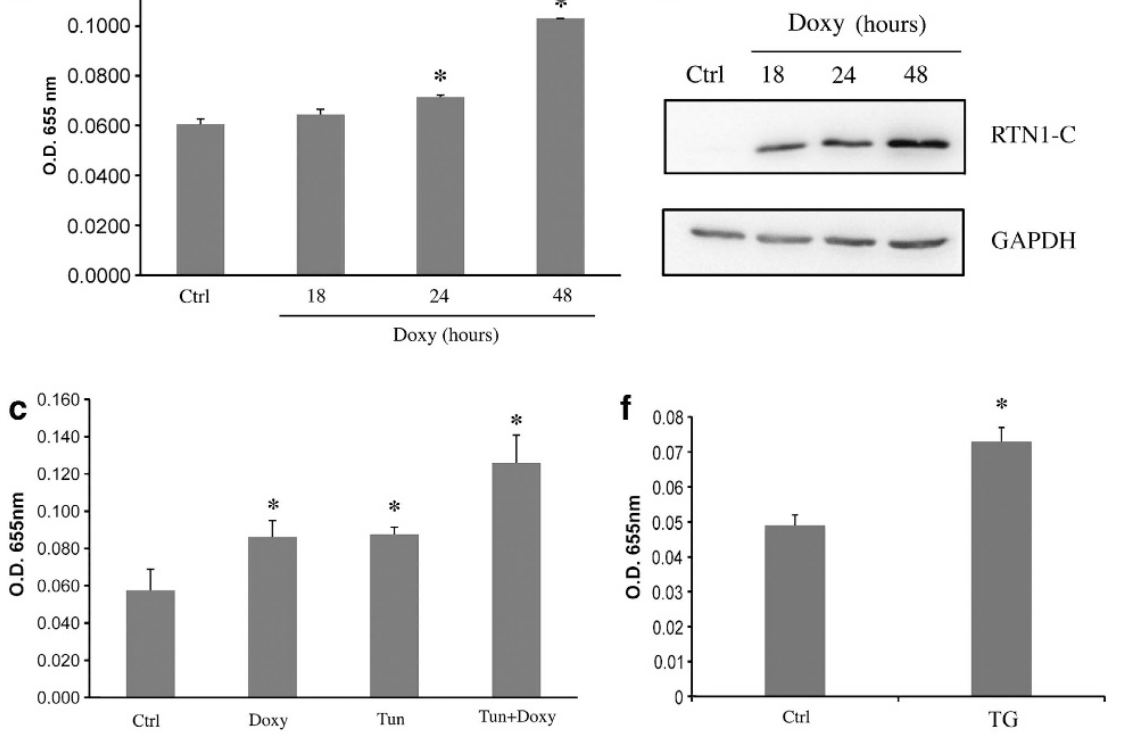

d

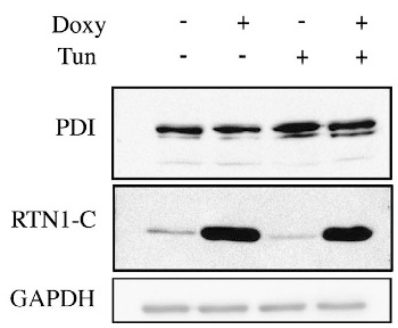

g
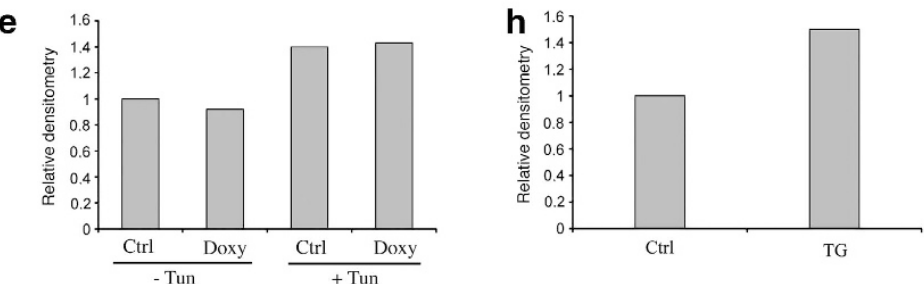

Figure 3 RTN1-C modulates PDI enzymatic activity. SH-SY5Y ${ }^{\text {RTN1-C }}$ cells were treated with $1 \mu \mathrm{g} / \mathrm{ml}$ doxycycline (Doxy) for 18,24 and $48 \mathrm{~h}$ and used to measure PDI enzymatic activity. (a) $80 \mu \mathrm{g}$ of total proteins were incubated with $0.16 \mathrm{mM}$ insulin and $1 \mathrm{mM}$ DTT read at $655 \mathrm{~nm}$ after $30 \mathrm{~min}$. Results represent the means \pm S.D. of three independent determinations. *Student's $t$-test, $P<0.01$ for each condition versus control. (b) Alternatively, cells were analyzed for RTN1-C expression by western blot. GAPDH was used as loading control. The results shown are representative of three independent experiments. (c and d) SH-SY5YRT1-C were treated with $1 \mu \mathrm{g} / \mathrm{ml}$ Doxy for $48 \mathrm{~h}$ or not (ctrl) in the presence or the absence of $5 \mu \mathrm{g} / \mathrm{ml}$ Tun for $24 \mathrm{~h}$ and analyzed by PDI activity assay (c) or western blot analysis (d) using anti-PDI, anti-RTN1-C and antiGAPDH (loading control) antibodies. The results shown are representative of three independent experiments. *Student's $t$-test, $P<0.01$ for each condition versus control. (e) Densitometric analysis of PDI protein levels relative to GAPDH. (f) SH-SY5YRTN1-C control cells were used to measure PDI enzymatic activity in the presence or the absence of $10 \mu \mathrm{M} \mathrm{TG}$ for $24 \mathrm{~h}$. The results shown are representative of three independent experiments. ${ }^{*}$ Student's $t$-test, $P<0.01$ versus control. (g) Western blot analysis of SH-SY5YRTN1-C control cells treated or not with $10 \mu \mathrm{M}$ TG for $24 \mathrm{~h}$ using anti-PDI and anti-GAPDH (loading control) antibodies. (h) Densitometric analysis of PDI protein levels relative to GAPDH. The results shown are representative of three independent experiments

\section{Discussion}

In this study, we have investigated the biological effects of RTN1-C, a member of the RTN family proteins, on PDI, in cells from neuronal origin.

We first demonstrated that RTN1-C is able to induce a dramatic cellular redistribution of PDI from a diffuse expression pattern to a puncuated localization. Importantly, we demonstrated that this phenomenon is tightly correlated to a significant increase of PDI enzymatic activity, with the formation of PDI puncta structures being rate limiting for its activity. Although it has been suggested that PDI is upregulated upon induction of ER stress, no direct evidence of a specific increase of its enzymatic activity has not been provided yet in these conditions. On the basis of data present in the literature to date, it has been speculated ${ }^{21}$ that RTN-mediated redistribution could lead to an increase in PDi activity. Importantly, here, we demonstrated for the first time that the cellular redistribution of PDI is associated with a significant rise in its activity. 

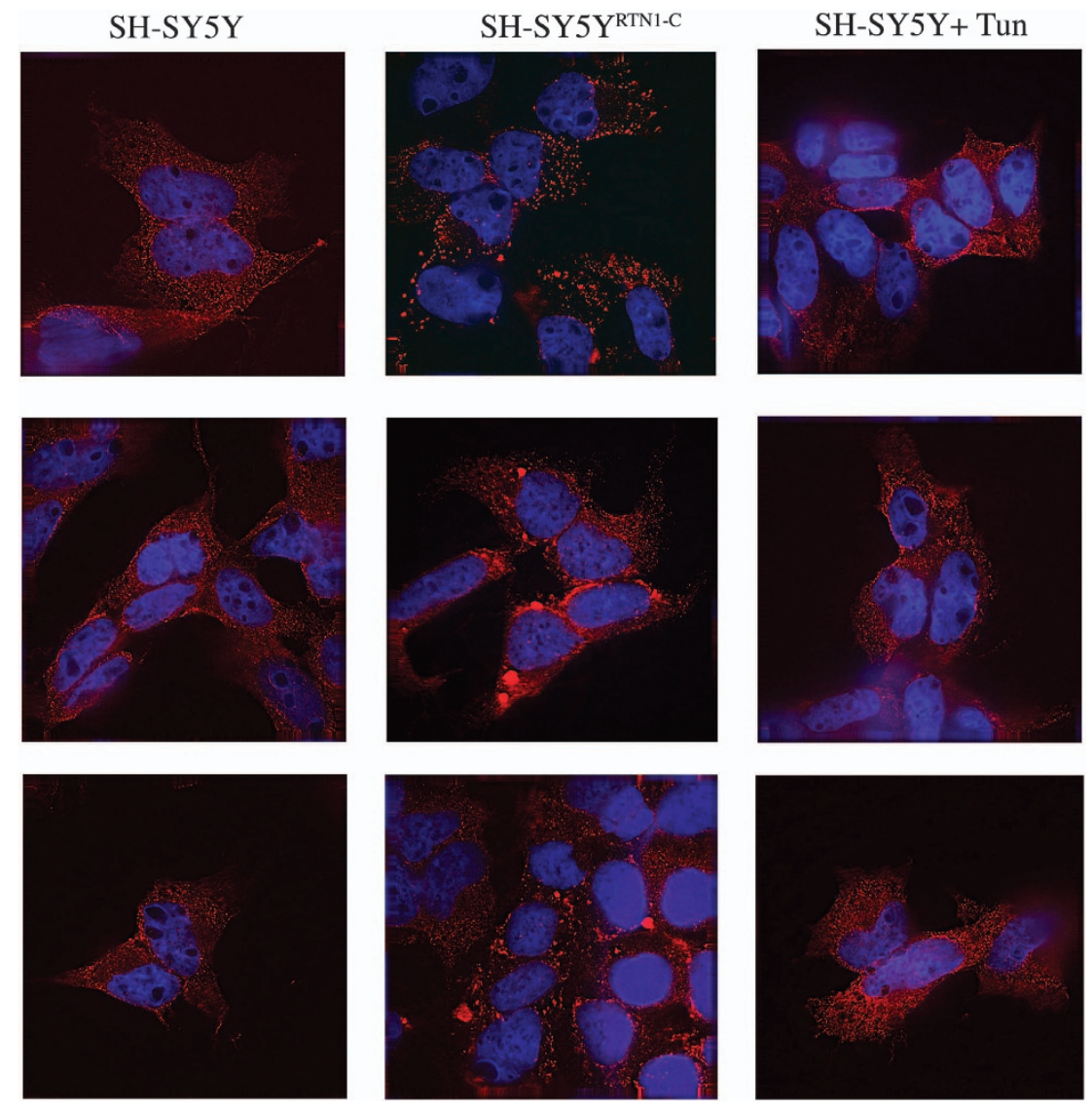

Figure 4 ER stress is not involved in PDI subcellular redistribution. Cells were treated with $1 \mu \mathrm{g} / \mathrm{ml} \mathrm{Doxy} \mathrm{or} 5 \mu \mathrm{g} / \mathrm{ml}$ Tun for $24 \mathrm{~h}$, stained with anti-PDI (red) antibodies and Hoechst 33342 and analyzed by fluorescence microscopy. Original magnifications: $\times 100$

In our previous works, we demonstrated that the upregulation of RTN1-C expression results in ER stress induction, finally leading to apoptotic cell death. ${ }^{14}$ On the basis of such evidence, we predicted that RTN1-C-mediated cell death could be the result of UPR attenuation and, consequently, a negative modulation of PDI activity. Indeed, it is generally accepted that, at an early stage, cell responds to ER stress induction by activating important cellular defences against protein misfolding, including PDI isomerase activity. Conversely, when the stress becomes severe and prolonged, these fine regulatory mechanisms are not sufficient and the cell undergoes apoptosis. ${ }^{22}$ Accordingly, it has been demonstrated that PDI overexpression results in both ER stress and ER stress-mediated apoptotic signaling attenuation. ${ }^{3}$ The results reported here indicate that the modulation of PDI activity by RTN1-C is an event independent of ER-mediated apoptosis. In fact, although we found that cellular treatment with well-known ER stress inducers (that is, tunycamicin and TG) is accompanied by an increase in PDI activity, no PDI cellular redistribution and puncta formation is induced. On the other hand, RTN1-C is able to modulate PDI activity without affecting its gene expression.

In order to investigate the mechanism by which RTN1-C affects PDI cellular localization and activity, we decided to use different RTN1-C mutants with the aim of detecting the specific domain of the protein involved in the observed phenomena. We previously demonstrated that the lysine 204 of RTN1-C is post-translationally modified by acetylation and this event is essential for inducing ER stress and cell death. According to our previous observation, the mutated isoform is still able to induce PDI redistribution and to modulate its enzymatic activity, further supporting the notion that these effects are independent of the RTN's role in cell death induction. Therefore, we hypothesize that the modulation of PDI activity could depend on other uncharacterized RTN-mediated function. We demonstrated that the $\mathrm{N}$-terminal region of RTN1-C is essential to affect PDI localization and activity; notably, this region has been demonstrated to regulate vesicle trafficking via the interaction with different components of SNARE complexes. ${ }^{10}$ Steiner et al. ${ }^{10}$ also showed that the N-terminal domain of RTN1-C is directly involved in this interaction. Thus, on the basis of these evidence, a possible explanation for RTN1-C-mediated PDI redistribution could be found in the well-known RTN involvement in membrane shaping and cellular trafficking. RTNs expression is known to affect ER morphology and function and, in particular, we previously demonstrated that RTN1-C expression causes dramatic changes in the ER structures. ${ }^{14}$ In this work we demonstrated that PDI-containing structures are ER-derived vesicles. On this basis it could be speculated that RTN1-C regulates PDI localization because of its 

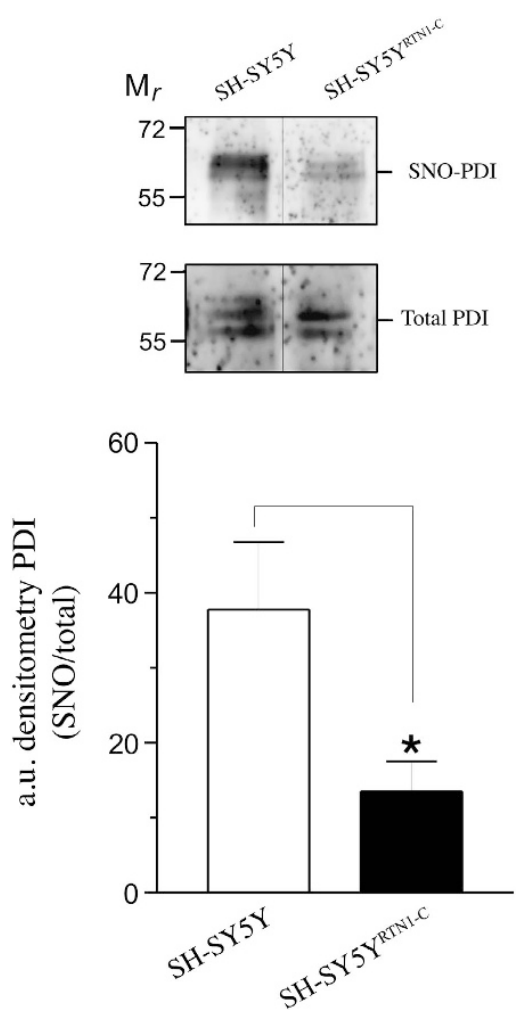

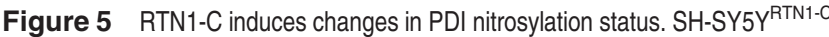
cells were treated with $1 \mu \mathrm{g} / \mathrm{ml}$ Doxy for $48 \mathrm{~h}$ and subjected to SNO-biotin-switch assay. Equal volumes of biotinylated proteins obtained from SH-SY5Y ${ }^{\text {RTN1-C }}$ cells and parental SH-SY5Y were let react with Sepharose-conjugated Streptavidin beads, pulled down and analyzed by western blot to detect SNO-PDI. Total PDI was used as control. Western blots shown are from one experiment representative of three that gave similar results. Densitometric analyses of SNO-PDI and total PDI were carried out using Quantity One Software (Bio-Rad). Data are expressed as SNO-PDI/total PDI ratio and represent the means \pm S.D. ${ }^{*} P<0.05$

role in regulating ER morphology and microtubule-based intracellular transport processes. It is very interesting that these cellular functions are often impaired in many neurodegenerative diseases. ${ }^{21}$ This assumption is supported by the findings that disruption of microtubule organization prevents PDI cellular redistribution.

PDI activity is regulated by post-translational modification through $S$-nitrosylation reactions at the catalytic cysteines of the active site, which impairs PDI activity. Interestingly, a number of recent studies established a functional relationship between the inhibition by $S$-nitrosylation of PDI, defects in regulation of protein folding within the $\mathrm{ER}$, and neurodegeneration. Analyses of human brains from Parkinson's disease (PD) or Alzheimer's patients support a causative role for PDI S-nytrosylation and ER stress in the onset of these neurodegenerative disorders. ${ }^{23,24}$ Such evidence suggests new insight into the role of aberrant $S$-nitrosylation in the pathogenesis of neurodegeneration and provides a valid rationale for novel and selective therapeutic approaches. ${ }^{25}$ For example, it has been reported that a small molecule mimicking PDI active site protects against toxicity of mutant $\mathrm{Cu} / \mathrm{Zn}$ superoxide dismutase inclusion formation typical of $\mathrm{ALS}^{3}$ Moreover, it has been shown that in models of

\section{a}

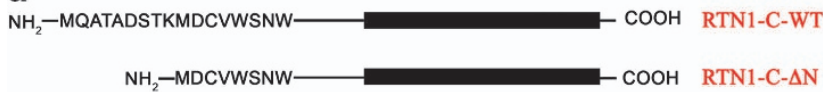

b

CTRL
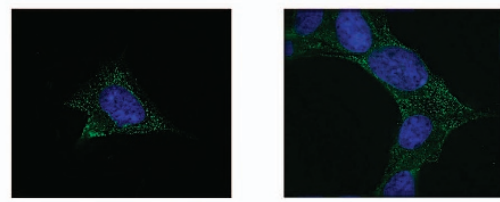

RTN1-C-WT
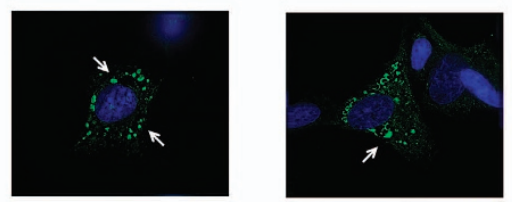

RTN1-C-K204
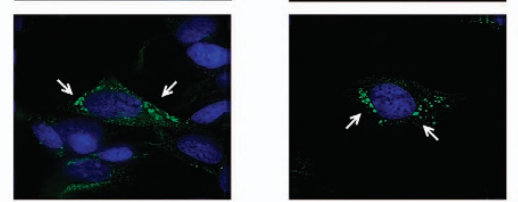

RTN1-C- $\Delta N$
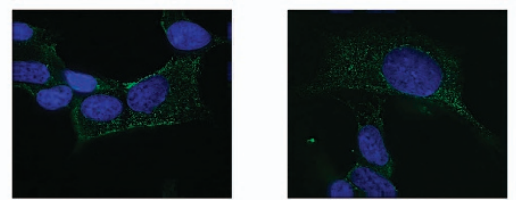

Figure 6 RTN1-C N-terminal domain is involved in PDI localization. (a) Schematic diagram showing RTN1-C mutant (RTN1-C- $\Delta$ N) generated by the deletion of the first nine amino acids at the $\mathrm{N}$-teminal region of the full-length isoform (RTN1-C-WT). (b) SH-SY5Y neuroblastoma cells were transfected for $48 \mathrm{~h}$ with an expression vector carrying the cDNA for wild-type (RTN1-C-WT) or different mutant form of RTN1-C (RTN1-C K204, RTN1-C- $\Delta$ N), or the empty vector, and analyzed by fluorescence microscopy. Cells were stained with anti-PDI (green) and nuclei were counterstained with Hoechst 33342 (blue). Images are representative of three independent experiments and show the different effects of RTN1-C mutant form on PDI distribution (arrows) compared with control cells

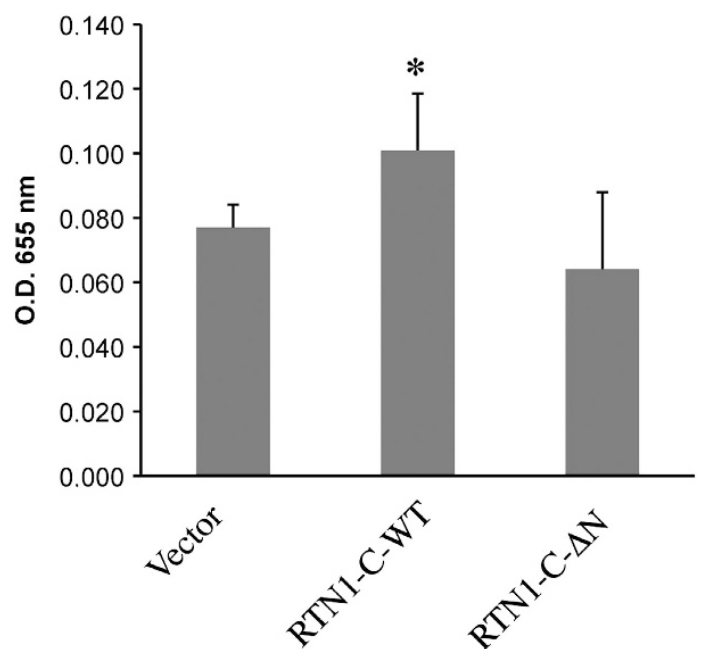

Figure 7 RTN1-C N-terminal mutant does not affect PDI activity. SH-SY5Y cells transfected for $48 \mathrm{~h}$ with an expression vector carrying the cDNA of wild-type RTN1C (RTN1-C-WT), the relative mutant form deleted for the first ten amino acids at the N-terminal region (RTN1-C- $\Delta \mathrm{N}$ ), or the vector alone (Ctrl). $80 \mathrm{~g}$ of total proteins were used to measure PDI activity as described in figure 3 . Results represent the means \pm S.D. of three different experiments. *Student's $t$-test, $P<0.01$ versus control 


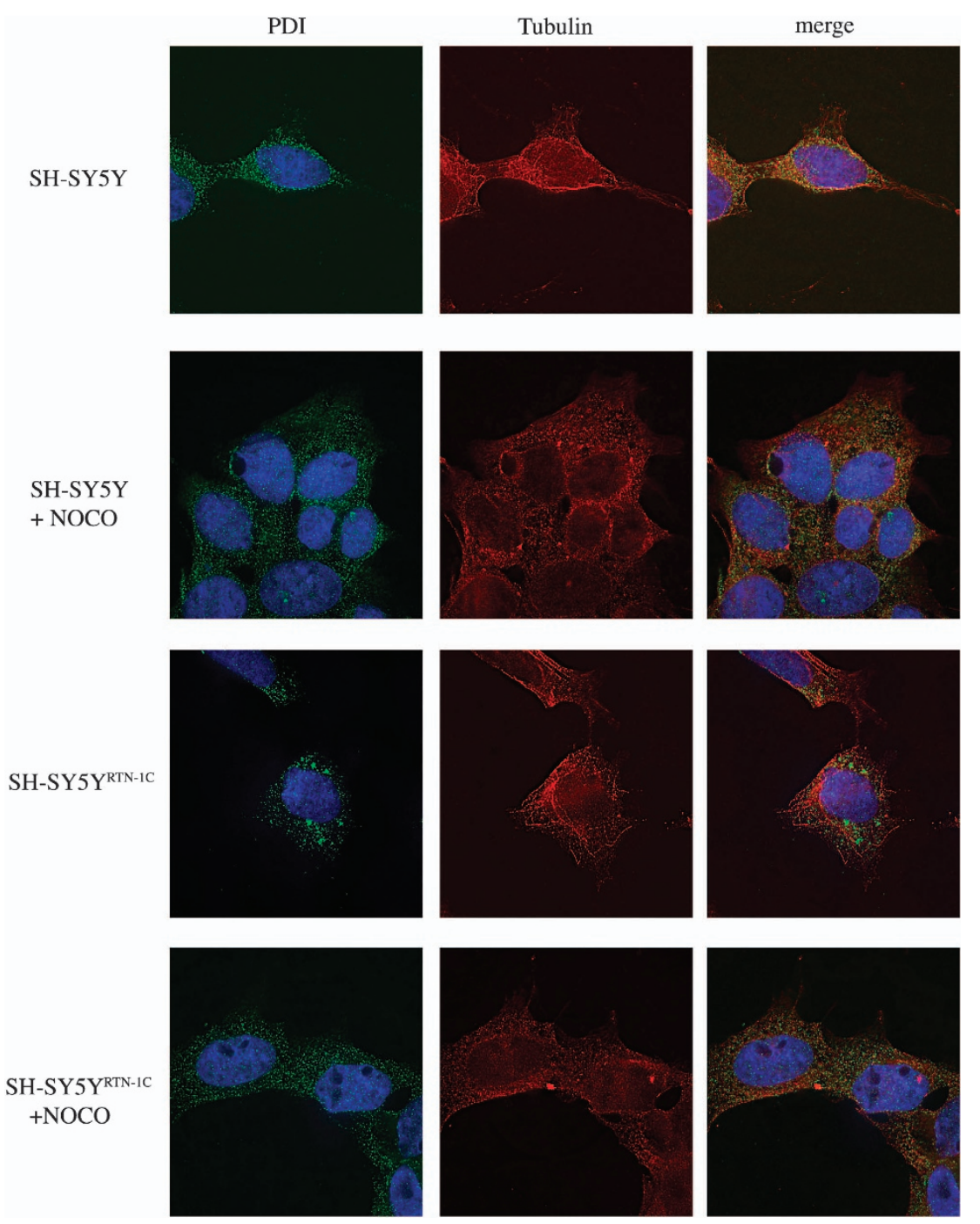

Figure 8 Microtubules structure is important for RTN1-C-mediated PDI redistribution. SH-SY5YRTN1-C cells were induced with $1 \mu \mathrm{g} / \mathrm{ml}$ Doxy for $48 \mathrm{~h}$; during the last $4 \mathrm{~h}$ of Doxy incubation, $10 \mu \mathrm{M}$ Noco were added to allow the disruption of microtubule organization. Fluorescence analysis was carried out by staining with anti-PDI (green) and antitubulin (red), whereas nuclei were labeled with Hoechst 33342 (blue). Images are representative of three different experiments

PD, PDI is mostly found $S$-nitrosylated. ${ }^{4}$ Thus the possibility of modulating $S$-nitrosylation events could open new possibilities in ameliorating aggregation and toxicity of mutated proteins observed in many different neurodegenerative disorders.

Here we have shown that modulation of RTN1-C expression is able to dramatically decrease the intracellular levels of SNO-PDI, thereby suggesting that RTN1-C is able to modulate nitrosative stress conditions. Various studies suggest that increased nitrosative stress causes protein $S$-nitrosylation, leading to protein aggregations, which are highly toxic to neurons and can promote neurodegeneration. ${ }^{26}$ In addition to inducing protein aggregation, recent studies show that nitrosative stress can also compromise a number of neuroprotective pathways by modifying the activity of many proteins through $S$-nitrosylation. ${ }^{27}$

Although the means by which the modulation of RTN1-C expression results in changes of nitrosative stress is currently unknown, it is significant that these results match our previous data demonstrating that RTN1-C is able to negatively modulate the expression of NOS enzyme. ${ }^{16}$ Taken together these findings indicate that RTN1-C is potentially a good candidate for the modulation of PDI function and are particularly relevant considering the emerging involvement of PDI as key chaperone in cellular defense against protein misfolding-characterized diseases.

\section{Materials and Methods}

Cell lines, transfections and treatments. Human neuroblastoma cell line SH-SY5Y was grown in 1:1 mixture of Dulbecco's modified Eagle's medium and Ham's F12 with Glutamax (Gibco, Paisley, UK), supplemented with $10 \%$ fetal bovine serum (FBS), $100 \mathrm{U} / \mathrm{ml}$ penicillin, and $100 \mu \mathrm{g} / \mathrm{ml}$ streptomycin, at $37^{\circ} \mathrm{C}$ in a humidified atmosphere of $5 \% \mathrm{CO}_{2}$ in air.

SH-SY5Y neuroblastoma cells carrying a tetracycline-regulated RTN1-C expression system (SH-SY5Y ${ }^{\text {RTN1-C }}$ ) were previously obtained in our laboratory, ${ }^{14}$ and were maintained in the same growth conditions as SH-SY5Y except for the presence of $10 \%$ tetracycline-free FBS. 
For transient transfections, cells were approximately seeded at $80 \%$ confluence and transfected $24 \mathrm{~h}$ later with each expression vector by using lipofectamine 2000 (Invitrogen, Paisley, UK) according to the manufacturer's instructions.

The wt HA-tagged RTN1-C and the corresponding mutant form for the first nine amino acids (HA-RTN1-C- $\Delta \mathrm{N}$ ) were obtained from a cDNA human brain library by PCR amplification using specific primers:

RTN1-C-WT (FORWARD 5'-AACTTAAGCTTCGATGCAGGCCACTGCCGAT-3'; REVERSE $3^{\prime}$-CTAGTGGATCCTTAAGCGTAATCTGGAACATCGTATGGGTAC TCAGCGTGCCTCTTAGCGCC- $5^{\prime}$

RTN1-C- $\triangle$ N Mutant(FORWARD 5'-ATTCCAAGCTTATGGACTGTGTGGGA-3'; REVERSE 3'-CTAGTGGATCCTTAAGCGTAATCTGGAACATCGTATGGGTAC TCAGCGTGCCTCTTAGCGCC- $5^{\prime}$ )

Fragments were cloned into Hindlll-BamHI sites of pCDNA $3.1 \mathrm{Zeo}(+)$ (Clontech, Mountain View, CA, USA).

Doxy was used at $1 \mu \mathrm{g} / \mathrm{ml}$ concentration. Tun $(5 \mu \mathrm{g} / \mathrm{ml})$ or TG $(10 \mu \mathrm{M})$ were dissolved in dimethyl sulfoxide (DMSO) and added to culture media for the indicated times. Noco, dissolved in DMSO, was used at the concentration of $10 \mu \mathrm{M}$ and administrated to the cells $4 \mathrm{~h}$ prior their collection. Equal volume of DMSO $(<0,1 \%$ of culture volume) was used to treat control cells.

Electron microscopy. SH-SY5Y cells were fixed with $2.5 \%$ glutaraldehyde in $0.1 \mathrm{M}$ cacodylate buffer, $\mathrm{pH} 7.4$, for $45 \mathrm{~min}$ at $4{ }^{\circ} \mathrm{C}$, rinsed in buffer, postfixed in $1 \%$ $\mathrm{OsO}_{4}$ in $0.1 \mathrm{M}$ cacodylate buffer, $\mathrm{pH} 7.4$, dehydrated and embedded in Epon. For immunogold analysis $\mathrm{SH}-\mathrm{SY} 5 \mathrm{Y}$ cells were fixed in $2 \%$ freshly depolymerized paraformaldehyde and $0.2 \%$ glutaraldehyde in $0.1 \mathrm{M}$ cacodilate buffer, $\mathrm{pH} 7.4$ for $1 \mathrm{~h}$ at $4{ }^{\circ} \mathrm{C}$. Samples were rinsed in the same buffer, partially dehydrated and embedded in London Resin White (LR White, Agar Scientific Ltd., Stansted, UK). Ultrathin sections were processed for immunogold technique. Grids were preincubated with $10 \%$ normal goat serum in $10 \mathrm{mM}$ phosphate-buffered saline (PBS) containing $1 \%$ bovine serum albumin (BSA) and $0.13 \% \mathrm{NaN}_{3}$ (medium $\mathrm{A}$ ), for $15 \mathrm{~min}$ at room temperature. Sections were then incubated with primary antibody, mouse anti-PDI(1D3) (Enzo Life Sciences, Florence, Italy) diluted 1:100 in medium $\mathrm{A}$ for $1 \mathrm{~h}$ at room temperature. After rinsing in medium A containing 0.01\% Tween 20 (Merck, Darmstadt, Germany), sections were incubated in goat anti-mouse IgG conjugated to $15 \mathrm{~nm}$ colloidal gold (British BioCell Int., Cardiff, UK), diluted 1:30 in medium $\mathrm{A}$, containing fish gelatine, for $1 \mathrm{~h}$ at room temperature. The double immunolabeling has been performed incubating the PDI-labeled sections with rabbit polyclonal anti-calreticulin (Stressgen, Brussels, Belgium) followed by goat anti-rabbit IgG conjugated to $5 \mathrm{~nm}$ colloidal gold (British BioCell Int.), diluted $1: 30$ in medium $\mathrm{A}$, containing fish gelatine, for $1 \mathrm{~h}$ at room temperature.

Grids were thoroughly rinsed in distilled water, contrasted with aqueous $2 \%$ uranyl acetate for $20 \mathrm{~min}$ and photographed in a Zeiss EM 900 electron microscope, Zeiss, Jena, Germany.

Immunofluorescence. Cells were grown for $48 \mathrm{~h}$ on poly-L-lysine-coated sterile glass slides. After medium removal, cells were washed twice in PBS, thereafter fixed in $4 \%$ parafolmaldehyde-PBS for $10 \mathrm{~min}$, and then permeabilized for 10 min with $0.1 \%$ Triton $X-100$. Next, cells were rinsed three times with PBS and nonspecific binding sites were blocked in PBS $/ 10 \%$ FBS for $30 \mathrm{~min}$. Appropriate primary antibodies were added for $1 \mathrm{~h}$ dissolved in PBS/1\% FBS solution. We used anti-RTN1-C mouse (1:1000; Abcam, Cambridge, UK), anti-PDI mouse (1:500; Stressgene), anti-PDI rabbit (1:500; StressMark, Florence, Italy) and anti-tubulin (1:1000; Sigma, St Louis, MO, USA) antibodies. After three washes in PBS, cells were incubated for $30 \mathrm{~min}$ with the appropriate anti-mouse and anti-rabbit secondary antibodies (Alexa, Molecular Probes, Monza, Italy) diluted $1: 1000$ in PBS/1\% FBS. Finally, cell nuclei were stained with Hoechst 33342 (Molecular Probes), and cells examined with an image workstation DeltaVision (Applied Precision, Washington, WA, USA) Olympus (Olympus Southend om sea, Essex, UK) $1 \times 70$ microscope, unless otherwise indicated.

Western blot analysis. Cells were harvested and resuspended in a $50 \mathrm{mM}$ Tris- $\mathrm{HCl}$ buffer at $\mathrm{pH} 7.5$ containing $25 \mathrm{mM} \mathrm{KCl}$, and $5 \mathrm{mM} \mathrm{MgCl}_{2}$. Then samples were sonicated and protein quantification was carried out according to the Bradford method, using a Bio-rad protein assay solution (Bio-rad, MI, Italy) and BSA as standard. Protein extracts were separated by SDS-PAGE and transferred on nitrocellulose membrane. After blocking in PBS $/ 5 \%$ milk for $1 \mathrm{~h}$, membranes were probed with a specific primary antibody: mouse anti-RTN1-C (1:1000; Abcam), mouse anti-PDI (1:1000; Stressgen), and rabbit anti-HA (1:2000; Sigma). Mouse anti-GAPDH (1:1000; Advanced ImmunoChemicals, MI, Italy) was used as loading control. The binding of primary antibodies were detected with secondary peroxidase-conjugated antibodies (1:5000; BD, Biosciences, San Jose, CA, USA), and visualized with the enhanced chemoluminescence system (Bio-rad) by using FluorChem SP machine (Alpha Innotech, Rondburg, South Africa).

PDI activity assay. PDI activity was measured according to Lovat et al. ${ }^{15}$ Cells were harvested, resuspended in a $50 \mathrm{mM}, \mathrm{pH} 7.5$ Tris- $\mathrm{HCl}$ buffer containing $25 \mathrm{mM} \mathrm{KCl}$, and $5 \mathrm{mM} \mathrm{MgCl}_{2}$ and sonicated. After protein quantification, $80 \mu \mathrm{g}$ of protein extracts were added in $200 \mu$ l of reaction mix containing $100 \mathrm{mM}, \mathrm{pH} 7.0$ potassium phosphate, $2 \mathrm{mM}$, pH 7.5 EDTA, $0.16 \mathrm{mM}$ bovine insulin (Sigma) and $1 \mathrm{mM}$ DTT. After $30 \mathrm{~min}$ of incubation at RT, the PDI activity was monitored as increased turbidity at $655 \mathrm{~nm}$. For every PDI activity assay, final values were determined by subtracting the background value, obtained by measuring the absorbance of a protein extract-free control, from the mean \pm S.D. resulting from the reading of two independent samples.

Biotin-switch assay and PDI-SNO detection. The biotin-switch assay was performed as described by Jaffrey et al. ${ }^{28}$ with minor modifications as follows. Cell pellets were homogenized in $200 \mu$ of HENC buffer (250 mM HEPES, pH 7.7, $1 \mathrm{mM}$ EDTA and $0.1 \mathrm{mM}$ neocuproine, $0.4 \% 3$-[(3-cholamidopropyl)dimethylammonio]-1-propanesulfonate). After $30 \mathrm{~min}$ incubation on ice, lysates were centrifuged for $30 \mathrm{~min}$ at 17000 r.p.m. at $4{ }^{\circ} \mathrm{C}$. Four volumes of blocking buffer (HEN buffer, 2,5\% SDS, 25 mM methyl methanethiosulfonate, MMTS) were added to the supernatant (adjusted to $1 \mathrm{mg}$ protein per $\mathrm{ml}$ ) and maintained in shaking at $50^{\circ} \mathrm{C}$ for $30 \mathrm{~min}$. After MMTS removal by three cycles of washing/precipitation in cold acetone, pellets were resuspended in HENS buffer (250 mM HEPES, pH 7.7, $0.1 \mathrm{mM}$ EDTA, $10 \mathrm{mM}$ neocuproine and $1 \%$ SDS). $0.2 \mathrm{mM} \mathrm{N}$-[6-(biotinamido)hexyll-3'-(2-pyridyldithio)propionamide (Biotin-HPDP, Pierce, Bradenton, FL, USA) and $5 \mathrm{mM}$ sodium ascorbate were then added to the solution and incubated in the dark for $1-3 \mathrm{~h}$, after which the remaining biotin-HPDP was removed by repeated precipitations in pre-chilled acetone.

Biotinylated proteins were incubated for $1 \mathrm{~h}$ with equal volume of StreptavidinSepharose high performance beads (GE Healthcare, Piscataway, NJ, USA), purified by repeated precipitations in Binding buffer and eluted with $100 \mathrm{mM}$ 2mercaptoethanol. Upon clarification, proteins were subjected to western blot analyses carried out with an anti-PDI antibody to detect SNO-PDI. Total PDI was used as control.

\section{Conflict of Interest}

The authors declare no conflict of interest.

Acknowledgements. We thank P. Mattioli for expert assistance and help with images processing. This work was supported by grants from, Compagnia di San Paolo, the Ministry of Health of Italy 'Ricerca Corrente' and 'Ricerca Finalizzata', the Ministry of University Research 'FIRB' and AIRC to F.D. The support of the EU grant 'Transpath' Marie Curie project is also acknowledged.

1. Puig A, Primm TP, Surendran R, Lee JC, Ballard KD, Orkiszewski RS et al. A 21-kDa $\mathrm{C}$-terminal fragment of protein-disulfide isomerase has isomerase, chaperone, and antichaperone activities. J Biol Chem 1997; 272: 32988-32994.

2. Benham AM. The protein disulfide isomerase family: key players in health and disease. Antioxid Redox Signal 2012; 16: 781-789.

3. Walker AK, Farg MA, Bye CR, McLean CA, Horne MK, Atkin JD. Protein disulphide isomerase protects against protein aggregation and is S-nitrosylated in amyotrophic lateral sclerosis. Brain 2010; 113(Pt 1): 105-116.

4. Uehara T, Nakamura T, Yao D, Shi ZQ, Gu Z, Ma Y et al. S-nitrosylated protein-disulphide isomerase links protein misfolding to neurodegeneration. Nature 2006; 441: 513-517.

5. Honjo $\mathrm{Y}$, Ito $\mathrm{H}$, Horibe $\mathrm{T}$, Takahashi $\mathrm{R}$, Kawakami K. Protein disulfide isomeraseimmunopositive inclusions in patients with Alzheimer disease. Brain Res 2010; 1349: 90-96.

6. Yang YS, Harel NY, Strittmatter SM. Reticulon-4A (Nogo-A) redistributes protein disulfide isomerase to protect mice from SOD1-dependent amyotrophic lateral sclerosis. J Neurosci 2009; 29: 13850-13859.

7. Oertle $\mathrm{T}$, Huber $\mathrm{C}$, van der Putten $\mathrm{H}$, Schwab ME. Genomic structure and functional characterisation of the promoters of human and mouse nogo/rtn4. J Mol Biol 2003; 325: 299-323.

8. Yang YS, Strittmatter SM. The reticulons: a family of proteins with diverse functions. Genome Biol 2007; 8: 234 
9. Iwahashi J, Hamada N. Human reticulon 1-A and 1-B interact with a medium chain of the AP-2 adaptor complex. Cell Mol Biol 2003 (Noisy-le-grand). 49 Online Pub:OL467-71.

10. Steiner P, Kulangara K, Sarria JC, Glauser L, Regazzi R, Hirling H. Reticulon $1-\mathrm{C} /$ neuroendocrine-specific protein-C interacts with SNARE proteins. J Neurochem 2004; 3: 569-580.

11. Gil V, Nicolas O, Mingorance A, Ureña JM, Tang BL, Hirata T et al. Nogo-A expression in the human hippocampus in normal aging and in Alzheimer disease. J Neuropathol Exp Neurol 2006; 65: 433-444.

12. Murayama KS, Kametani F, Saito S, Kume H, Akiyama H, Araki W. Reticulons RTN3 and RTN4-B/C interact with BACE1 and inhibit its ability to produce amyloid beta-protein. Eur $J$ Neurosci 2006; 24: 1237-1244.

13. Yan R, Shi $Q, H u X$, Zhou X. Reticulon proteins: emerging players in neurodegenerative diseases. Cell Mol Life Sci 2006; 63: 877-889.

14. Di Sano F, Fazi B, Tufi R, Nardacci R, Piacentini M. Reticulon-1C acts as a molecular switch between endoplasmic reticulum stress and genotoxic cell death pathway in human neuroblastoma cells. J Neurochem 2007; 102: 345-353.

15. Lovat PE, Corazzari M, Armstrong JL, Martin S, Pagliarini V, Hill D et al. Increasing melanoma cell death using inhibitors of protein disulfide isomerases to abrogate survival responses to endoplasmic reticulum stress. Cancer Res 2008; 68: 5363-5369.

16. Fazi $B$, Biancolella $M$, Mehdawy $B$, Corazzari $M$, Minella $D$, Blandini $F$ et al. Characterization of gene expression induced by RTN-1C in human neuroblastoma cells and in mouse brain. Neurobiol Dis 2010; 40: 634-644.

17. Tagami S, Eguchi $Y$, Kinoshita M, Takeda M, Tsujimoto Y. A novel protein, RTN-XS, interacts with both $\mathrm{Bcl}-\mathrm{XL}$ and $\mathrm{Bcl}-2$ on endoplasmic reticulum and reduces their antiapoptotic activity. Oncogene 2000; 19: 5736-5746.

18. He W, Lu Y, Qahwash I, Hu XY, Chang A, Yan R. Reticulon family members modulate BACE1 activity and amyloid-beta peptide generation. Nat Med 2004; 10: 959-965.

19. Fazi B, Melino S, De Rubeis S, Bagni C, Paci M, Piacentini M et al. Acetylation of RTN1-C regulates the induction of ER stress by the inhibition of HDAC activity in neuroectodermal tumors. Oncogene 2009; 28: 3814-3824.
20. Pokrywka NJ, Payne-Tobin A, Raley-Susman KM, Swartzman S. Microtubules, the ER and Exu: new associations revealed by analysis of mini spindles mutations. Mech Dev 2009; 126: 289-300.

21. Walker AK. Protein disulfide isomerase and the endoplasmic reticulum in amyotrophic lateral sclerosis. J Neurosci 2010; 30: 3865-3867.

22. Urra $\mathrm{H}$, Hetz $\mathrm{C}$. The ER in $4 \mathrm{D}$ : a novel stress pathway controlling endoplasmic reticulum membrane remodeling. Cell Death Differ 2012; 19: 1893-1895.

23. Nakamura T, Lipton SA. Redox modulation by S-nitrosylation contributes to protein misfolding, mitochondrial dynamics, and neuronal synaptic damage in neurodegenerative diseases. Cell Death Differ 2011; 18: 1478-1486.

24. Benhar M, Forrester MT, Stamler JS. Nitrosative stress in the ER: new role for S-nitrosylation in neurodegenerative diseases. ACS Chem Biol 2006; 1 : 355-358.

25. Haldar SM, Stamler JS. S-Nitrosylation at the interface of autophagy and disease. Mol Cell 2011; 43: 1-3.

26. Gu Z, Nakamura T, Lipton SA. Redox reactions induced by nitrosative stress mediate protein misfolding and mitochondrial dysfunction in neurodegenerative diseases. $\mathrm{Mol}$ Neurobiol 2010; 41: 55-72.

27. Chung KK, David KK. Emerging roles of nitric oxide in neurodegeneration. Nitric Oxide 2010; 22: 290-295.

28. Jaffrey SR, Snyder SH. The biotin switch method for the detection of S-nitrosylated proteins. Sci STKE 2001; 2001: pl1.

(c) $\odot$ Cell Death and Disease is an open-access journal Cy ${ }_{\mathrm{BY}} \mathrm{ND}$ published by Nature Publishing Group. This work is licensed under a Creative Commons Attribution-NonCommercialNoDerivs 3.0 Unported License. To view a copy of this license, visit http://creativecommons.org/licenses/by-nc-nd/3.0/

\section{Supplementary Information accompanies this paper on Cell Death and Disease website (http://www.nature.com/cddis)}

